\title{
Circulating Long Non-Coding RNAs Act as Biomarkers for Predicting ${ }^{131}$ I Uptake and Mortality in Papillary Thyroid Cancer Patients with Lung Metastases
}

\author{
Zhong-Ling Qiu ${ }^{a} \quad$ Chen-Tian Shen ${ }^{a} \quad$ Zhen-kui Sun ${ }^{a}$ Wei-Jun Weia Xin-Yun Zhang ${ }^{a}$ \\ Hong-Jun Songa Quan-Yong Luo
}

aDepartment of Nuclear Medicine, Shanghai Jiao Tong University Affiliated Sixth People's Hospital, Shanghai, China

\section{Key Words}

Papillary thyroid cancer ${ }^{131}$ I therapy $\bullet$ plasma LncRNAs $\bullet$ Lung metastases

\begin{abstract}
Purpose: The aims of the current study were to explore plasma IncRNAs as a novel biomarker panel for the diagnosis of non-131 I-avid lung metastases of PTC and to investigate the plasma IncRNA expression levels associated with survival in PTC patients with lung metastases. Methods: The expression of IncRNAs was examined using an IncRNA microarray chip. The IncRNAs with the most significant difference in expression between PTC patients with non${ }^{131}$ I-avid lung metastases and PTC patients with ${ }^{131}$ I-avid lung metastases were verified by quantitative reverse-transcription polymerase chain reaction. The Kaplan-Meier method was used to determine whether the plasma IncRNA levels might be indicative of patient prognosis. Results: Compared with ${ }^{131}$ I-avid lung metastases, we discovered that two IncRNAs (ENST00000462717 andENST00000415582) were upregulated and two (TCONS_00024700 and NR_028494) were downregulated in the non-131I-avid lung metastases of PTC. Receiver operating characteristic curve (ROC) analyses indicated that the use of these four IncRNAs had high diagnostic sensitivity and specificity for predicting non- ${ }^{131}$ I-avid lung metastases of PTC. The merged areas under the curve for ENST00000462717, ENST00000415582, TCONS_00024700, and NR_028494 in the training and validation sets were $0.890,0.936,0.975$, and 0.918, respectively. Low (ENST00000462717 and ENST00000415582) and high plasma IncRNA levels(TCONS_00024700and NR_028494) were also found to be associated with better prognosis of PTC patients with lung metastases $(P<0.001)$. Conclusions: ENST00000462717, ENST00000415582, TCONS_00024700, and NR_028494 may be used as novel and minimally invasive markers for the diagnosis and prognostic assessment of non-131I-avid lung metastases from PTC.

Z.-L. Qiu, C.-T. Shen and Z.-K. Sun are joint first authors and contributed equally to this work. 


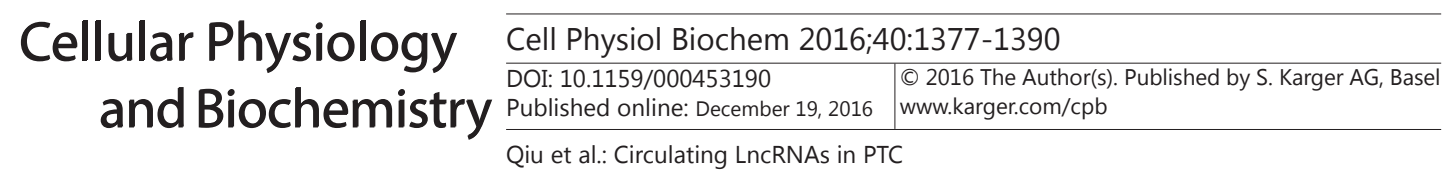

\section{Introduction}

Thyroid cancer was one of the cancers with the most rapidly increasing prevalence in the United States between 1975 and 2012, with an estimated 62,450 new cases in 2015 [1]. Its rising incidence has also been documented in many other countries [2]. Papillary thyroid carcinoma (PTC) is the most common type of thyroid cancer and accounts for $70 \%-90 \%$ of differentiated thyroid cancer (DTC). It typically involves an indolent tumor associated with a favorable prognosis, with ten survival rates of $~ 90 \%$ [3].

Metastasis to lung, the most common site of distant metastasis of DTC, is associated with a relatively poor prognosis [4]. Treatment of patients with lung metastasis is based on radioiodine $\left({ }^{131} \mathrm{I}\right.$ ) and associated with levothyroxine (T4) suppression [4]. Two-thirds of lung metastasis patients have ${ }^{131}$ I uptake on a therapeutic ${ }^{131}$ I whole-body scan $\left({ }^{131}\right.$ I-WBS), and a half of these achieve remission. The remaining one-third of such patients have no ${ }^{131}$ I uptake ability and die early because of ${ }^{131}$ I-refractory PTC $[4,5]$. Without ${ }^{131}$ I uptake, radioiodine therapy has no obvious benefit for those with non- ${ }^{131}$ I-avid metastatic lesions [6]. Such patients should not undergo unnecessary ${ }^{131}$ I treatment and can thus avoid the risk of high serum TSH stimulation after thyroxin withdrawal and the side effects of such treatment. Therefore, it is important to identify accurate predictive biomarkers in order to develop novel ${ }^{131}$ I therapeutic strategies. Specifically, promising noninvasive biomarkers to identify non- ${ }^{131}$ I-avid lung metastasis of PTC prior to ${ }^{131}$ I therapy are urgently needed. Compared with sampling of tissue, blood is minimally invasive to sample and easy to obtain, making it attractive to explore potential biomarkers.

Long noncoding RNAs (lncRNAs) are RNA molecules that are longer than 200 nucleotides and not translated into proteins [7]. Although these long noncoding transcripts were once considered to be simply transcriptional "noise" or cloning artifacts [8], recent evidence has shown that lncRNAs in PTC patients play important roles in diverse biological processes, such as transcriptional regulation, cell growth, tumorigenesis, and as novel targets for PTC diagnostics and therapeutics [9-15]. Interestingly, recent studies have suggested that IncRNAs are also present in serum, plasma, and other bodily fluids [16-18], and some plasma lncRNAs have been described as candidate biomarkers [19-21].

In this study, by using both Affymetrix (CA, USA) IncRNA microarrays and quantitative reverse-transcription polymerase chain reaction (qRT-PCR) assays, we aimed to explore the genome-wide IncRNA expression profile in plasma from non- ${ }^{-131} \mathrm{I}$-avid lung metastases compared with that in ${ }^{131}$ I-avid lung metastases from PTC, and thus sought to explore plasma lncRNAs that might serve as a novel biomarker panel in the diagnosis of non- ${ }^{-131}$ I-avid lung metastases of PTC. We also investigated whether plasma lncRNA expression level might be associated with survival in PTC patients with lung metastases.

\section{Materials and Methods}

Patient sample preparation

Venous blood samples were obtained from PTC patients just before ${ }^{131}$ I therapy at the Nuclear Medicine Department of Shanghai Jiao Tong University-affiliated Sixth People's Hospital, a major ${ }^{131}$ I treatment center in China. In total, 204 patients with lung metastases who had been pathologically diagnosed with PTC after total thyroidectomy with neck lymph node dissection between 2005 and 2015 were enrolled in the study. Three to five months after total thyroidectomy, each patient received an ablative dose of ${ }^{131}$ I and was put on a low iodine diet for 3-4 weeks. ${ }^{131}$ I-WBS was performed 3-5 days after the administration of ${ }^{131}$ I at a dosage of 150-200 mCi. The patients included in the study were divided into two age- and sex-matched groups: a non- ${ }^{131} \mathrm{I}$-avid lung metastasis group (group A) and a ${ }^{131} \mathrm{I}$-avid lung metastasis group (group B). A post-therapy patients showing no ${ }^{131}$ I uptake on the ${ }^{131}$ I-WBS (defined as a level of ${ }^{131}$ I uptake in the lung that was similar to that in the upper limbs) were assigned to group A and those with diffuse ${ }^{131}$ I uptake to group B. PTC lung metastases in the group A were diagnosed by elevated serum thyroglobulin (Tg) and lung computed tomography (CT); PTC lung metastases in group B were diagnosed by ${ }^{131}$ I-WBS, elevated serum Tg and lung CT. 


\section{Cellular Physiology Cell Physiol Biochem 2016;40:1377-1390 \begin{tabular}{l|l} 
and Biochemistry Published online: December 19, 2016 & $\begin{array}{l}\text { (c) } 2016 \text { The Author(s). Published by S. Karger AG, Basel } \\
\text { www.karger.com/cpb }\end{array}$
\end{tabular} \\ Qiu et al.: Circulating LncRNAs in PTC}

\section{RNA extraction}

Blood samples collected from each patient were placed in an EDTA anticoagulant tube. The plasma was separated by centrifugation at $800 \times \mathrm{g}$ for $10 \mathrm{~min}$ at room temperature, followed by 15 -min high-speed centrifugation at $10,000 \times \mathrm{g}$ at room temperature to completely remove cell debris. The supernatant plasma was recovered and stored at $-80^{\circ} \mathrm{C}$ until analysis. We extracted total RNA from $600 \mu \mathrm{L}$ of plasma using TRIzol reagent (Sigma), in accordance with the protocol of the manufacturer. RNA quantity and quality were confirmed using a Nano Drop ND-1000 spectrophotometer. RNA integrity and gDNA contamination were tested by denatured agarose gel electrophoresis.

\section{Microarray and computational analyses}

Five pairs of patients were chosen for the microarray analyses between non- ${ }^{131}$ I avid patients and ${ }^{131}$ I avid patients. The inclusion criteria of this five pairs of patients were as following: 1) Age, sex, height, weight, the size and number of lung metastases, and Tg level (as a marker for recurrence and metastasis of PTC related to the tumor burden) were approximately similar; 2) nationality, race and the TNM staging of the primary PTC were the same; 3) no other malignant tumor history.

Specimen labeling and microarray hybridization were completed using a modified version of the Agilent One-Color Microarray-Based Gene Expression Analysis Protocol (Agilent Technologies, Santa Clara, CA, USA). The sample preparation and microarray hybridization were performed in accordance with the manufacturer's instructions with minor modifications. In brief, mRNA was purified from $1 \mu \mathrm{g}$ of total RNA following the removal of rRNA using an mRNA-ONLYTM eukaryotic mRNA isolation kit (Epicentre Biotechnologies, Madison, WI, USA). Subsequently, each sample was amplified and transcribed into fluorescent cRNA along the entire length of the transcripts without $3^{\prime}$ bias using a random priming method. The labeled cRNAs were hybridized onto a Human lncRNA Array v3.0 (8×60 K; Arraystar, Rockville, MD, USA), designed for 30,586 lncRNAs and 26,109 coding transcripts. The IncRNAs were carefully constructed using the most highly respected public transcriptome databases, including Refseq (http://www.ncbi.nlm.nih.gov/ refseq/), UCSC Known Genes (http://www.biomedsearch.com/nih/UCSC-Known-Genes/16500937.html), and GENCODE (http://www.gencodegenes.org/), as well as landmark publications [22-24]. Each transcript was accurately identified by a specific exon or splice junction probe. Positive probes for housekeeping genes and negative probes were also printed onto the arrays for hybridization quality control. After washing the slides, the arrays were scanned using a G2505C scanner (Agilent Technologies Inc.), and the acquired images of the arrays were analyzed using Feature Extraction software (version 11.0.1.1; Agilent Technologies Inc.). Quantile normalization and subsequent data processing were performed using the GeneSpring GX v12.0 software package (Agilent Technologies Inc.). The microarray was performed by KangChen Bio-tech (Shanghai, China).

\section{Validation}

To validate the differentially expressed between PTC patients non- ${ }^{131} \mathrm{I}$-avid lung metastases and PTC patients ${ }^{131} \mathrm{I}$-avid lung metastases, the candidates were tested by qRT-PCR in an independent cohort from the patients remaining in group A and group B after the exclusion of the samples used in the microarray assays. Total RNA was extracted and purified using the miRNeasy Mini Kit (Qiagen, Valencia, CA, USA), in accordance with the manufacturer's instructions, and stored at $-80^{\circ} \mathrm{C}$. RevertAid ${ }^{\mathrm{TM}}$ First Strand cDNA Synthesis Kit (K1622; Fermentas) was used for reverse transcription. qRT-PCR was performed in the ABI PRISM 7500 Sequence Detection System (Applied Biosystems, Foster City, CA) using the SYBR Green RT-PCR kit (Qiagen, Hilden,Germany). All values were normalized using an internal reference (U6, for miRNAs)

Evaluation of ${ }^{131}$ I therapeutic efficacy in the ${ }^{131}$ I-avid lung metastases group

The therapeutic effects of ${ }^{131}$ I were evaluated on the basis of the size of the metastatic lung nodules using CT. Lung nodule size was assessed according to the Response Evaluation Criteria in Solid Tumors (RECIST v1.1) [25]: complete response (CR), disappearance of all target lesions or any pathological lesions (target or non-target) must have a reduction in short axis to $<10 \mathrm{~mm}$; partial response (PR), $\geq 30 \%$ decrease in the diameters of target lesions; progressive disease (PD), $\geq 20 \%$ increase in the diameters of target lesions combined with an absolute increase of $\geq 5 \mathrm{~mm}$ in the sum of diameters (the appearance of one or more new lesions was also considered progression); stable disease (SD), neither sufficient shrinkage to qualify for PR nor sufficient increase to qualify for PD. CR, PR and SD were defined as effectiveness and PD was defined as ineffectiveness for the ${ }^{131} \mathrm{I}$-avid lung metastases group. 


\section{Cellular Physiology Cell Physiol Biochem 2016;40:1377-1390

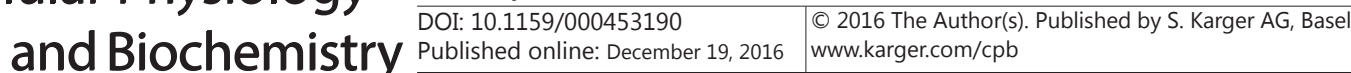 \\ Qiu et al.: Circulating LncRNAs in PTC}

Statistical analysis

The levels of IncRNAs differentially expressed between different groups were analyzed using Student's t-test with fold change $\geq 2.0$ and $p<0.05$ considered to indicate statistical significance. Comparisons of continuous variables between groups A and B were performed using Student's t-test, while categorical variables were analyzed using the chi-square test. Receiver operating characteristic (ROC) curves were constructed, and the area under the curve (AUC) was calculated to evaluate the specificity and sensitivity of predicting non- ${ }^{131} \mathrm{I}$-avid lung metastases. Ten-year survival rates were analyzed using the Kaplan-Meier method with the log-rank test used for comparisons between group A and B. MedCalc software version 17.0 (MedCalc, Mariakerke, Belgium) and SPSS17.0 (SPSS Inc., Chicago, IL, USA) were used for statistical analyses.

\section{Results}

\section{Patient characteristics}

The results of the analysis of clinicopathological relevance (total of 204 patients) are summarized in Table 1. Among these 204 patients, 71 were assigned to group A and 133 to group B. These two groups had no significant differences in parameters such as age and gender " $(\mathrm{p}>0.05)$ ". No other distant metastatic sites were identified. A positive history of other tumors, other treatment and other subtypes of PTC were ruled out.

\section{Microarray-based detection of plasma IncRNAs}

Human lncRNA Array (version 3.0; Agilent) was applied to detect the IncRNAs derived from the plasma of five PTC patients with non- ${ }^{131}$ I-avid lung metastases from group A, and five PTC patients with ${ }^{131} \mathrm{I}$-avid lung metastases from group B. Compared with the ${ }^{131} \mathrm{I}$-avid lung metastases, we found that there were $1006 \mathrm{lncRNAs}$ that were upregulated (fold change $\geq 2.0$ and $\mathrm{P}<0.05$ ) and 705 that were downregulated(fold change $\geq 2.0$ and $\mathrm{P}<0.05$ ) in non${ }^{131}$ I-avid lung metastases, as shown in Fig. 1 . To screen the biomarkers for their ability to predict non- ${ }^{131}$ I-avid lung metastases of PTC, fold change $\geq 10$ and false discovery rate (FDR) $<0.01$ were used as the cutoff. Compared with the lncRNAs in ${ }^{131}$ I-avid lung metastases, we identified four upregulated IncRNAs and 10 downregulated ones in the non-131I-avid lung metastasis group (Fig. 2). Fourteen candidate lncRNAs were selected to be validated in 20 pairs of samples in the following training set (Table 2).

Table. 1. Clinical and demographic features of patients with non-131Iavid (group A) and 131I-avid lung metastases (group B) from PTC

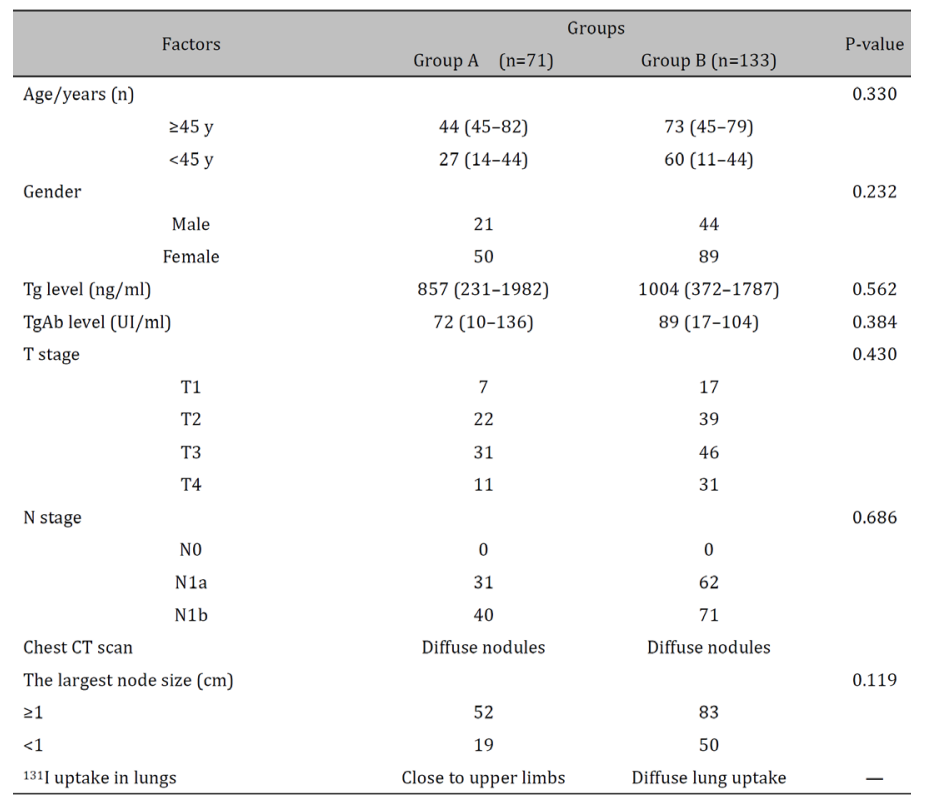


Table. 2. Significantly differentially expressed lncRNAs ENST00000415582 between group NR_033989

A and group B as ENST00000439588 determined by ENST00000415869 microarray (fold TCONS_00024700 change $\geq 10$ and ENST00000570512 FDR $<0.01$ )

\begin{tabular}{|c|c|c|c|c|c|c|}
\hline Seqname & Chromosome & Strand & $\begin{array}{l}\text { Regulation } \\
(\mathrm{A} / \mathrm{B})\end{array}$ & Fold change & FDR & $\mathrm{p}$-value \\
\hline ENST00000462717 & chr3 & + & up & 74.0481414 & 0.005173002 & 8.02823E-06 \\
\hline ENST00000415582 & $\operatorname{chr} 1$ & - & up & 36.3585354 & 0.005505494 & $9.74059 \mathrm{E}-06$ \\
\hline NR_033989 & chr11 & - & up & 11.0530831 & 0.000184118 & $4.69249 \mathrm{E}-08$ \\
\hline ENST00000439588 & $\operatorname{chr} 22$ & + & up & 10.7172302 & 0.000847474 & $5.03978 \mathrm{E}-07$ \\
\hline ENST00000415869 & $\operatorname{chr} 3$ & - & down & 268.305597 & 0.005642146 & $1.05452 \mathrm{E}-05$ \\
\hline TCONS_00024700 & chr16 & - & down & 66.9496153 & 0.000117948 & $1.71981 \mathrm{E}-08$ \\
\hline ENST00000570512 & chr17 & + & down & 45.7143709 & 0.00325372 & $4.42269 \mathrm{E}-06$ \\
\hline HMlincRNA284+ & chr2 & + & down & 34.6902973 & 0.00320032 & $3.64491 \mathrm{E}-06$ \\
\hline NR_028494 & chr6 & - & down & 23.824772 & 0.000117948 & $2.00404 \mathrm{E}-08$ \\
\hline chr14:84031800-84050525+ & chr14 & + & down & 17.278456 & 0.001318668 & $8.96215 \mathrm{E}-07$ \\
\hline ENST00000581541 & chr18 & - & down & 14.606191 & 0.008202495 & $1.95115 \mathrm{E}-05$ \\
\hline ENST00000507733 & $\operatorname{chr} 5$ & - & down & 14.4649268 & 0.007680034 & $1.76163 \mathrm{E}-05$ \\
\hline TCONS_00026676 & $\operatorname{chr} 18$ & - & down & 11.9257492 & 0.000204055 & $6.93415 \mathrm{E}-08$ \\
\hline ENST00000445865 & chr2 & - & down & 11.2516248 & 0.000364332 & $1.8571 \mathrm{E}-07$ \\
\hline
\end{tabular}

Fig. 1. Volcano plot shows the variation of lncRNAs between the non- ${ }^{131}$ I-avid lung metastasis group (five patients from group A) and the ${ }^{131}$ I-avid lung metastasis group (five patients from group B). The vertical blue lines correspond to 2.0-fold increases and decreases, and the blue horizontal line represents $\mathrm{P}=0.05$. Thus, the red points in the plot represent the IncRNAs that were differentially expressed with statistical significance (A). Scatter plots are the normalized signal values of the samples (log2-scaled). The lncRNAs above the top green line and below the bottom green line indicate more than 2.0 -fold difference
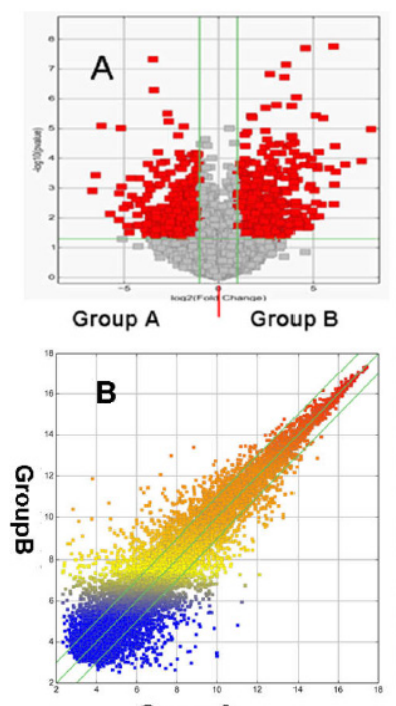

Group A

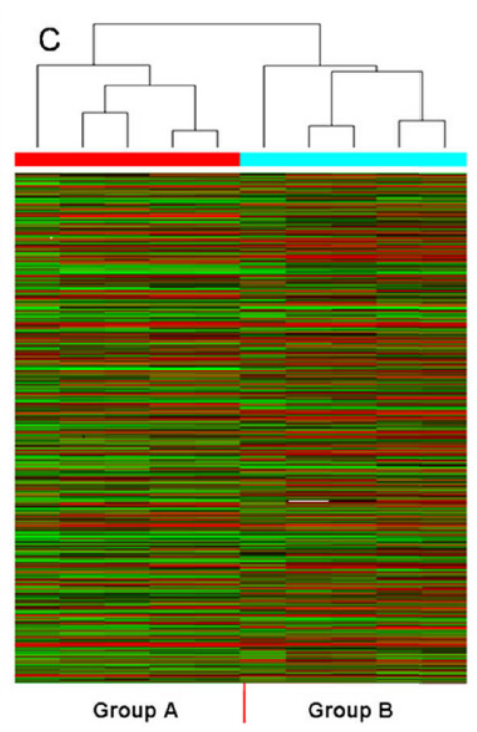

of IncRNA expression between group A and group B (B). The hierarchical clustering was performed based on IncRNAs differentially expressed between group A and group B. The results from the hierarchical clustering show IncRNA expression profiles that are distinguishable between group A and group B (C).

Fig. 2. Column diagram of four upregulated lncRNAs and 10 downregulated lncRNAs in group $A$ by microarray analyses (fold change $\geq 10$ and $\mathrm{PDR}<0.01$ were used as the cutoff).

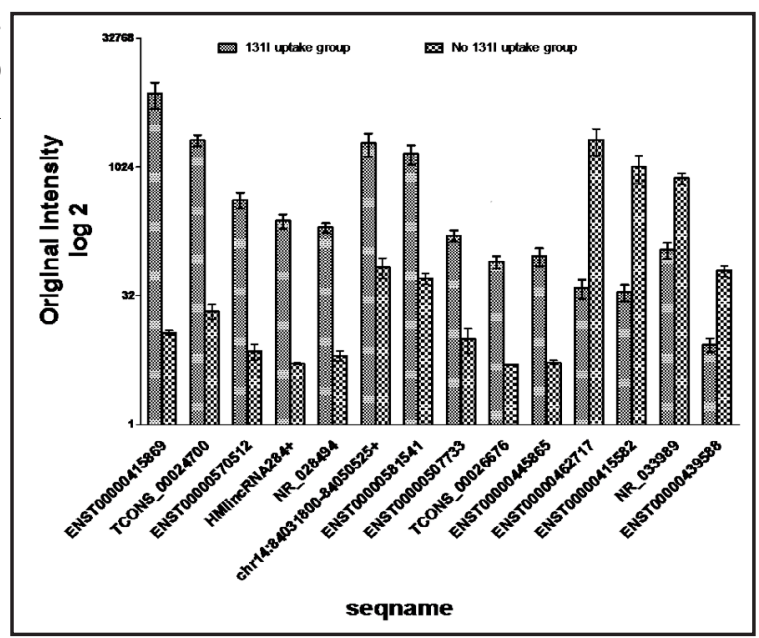


Fig. 3. A total of 20 plasma samples paired between group A and group B were used for validating the expression of four upregulated candidate IncRNAs in group A during qRT-PCR analysis. Data are presented as mean \pm SEM. Data was analyzed using Student's t-test. ${ }^{* *}, \mathrm{p}<0.05$.

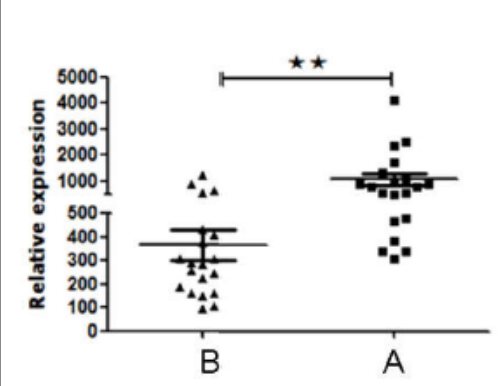

ENST00000462717

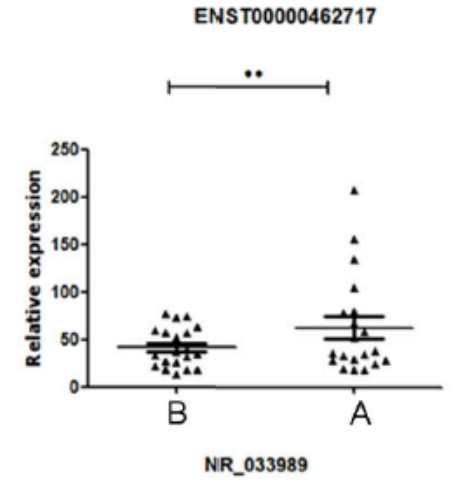

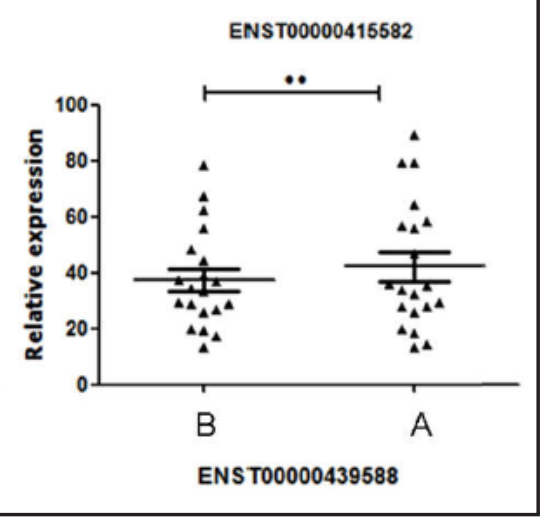

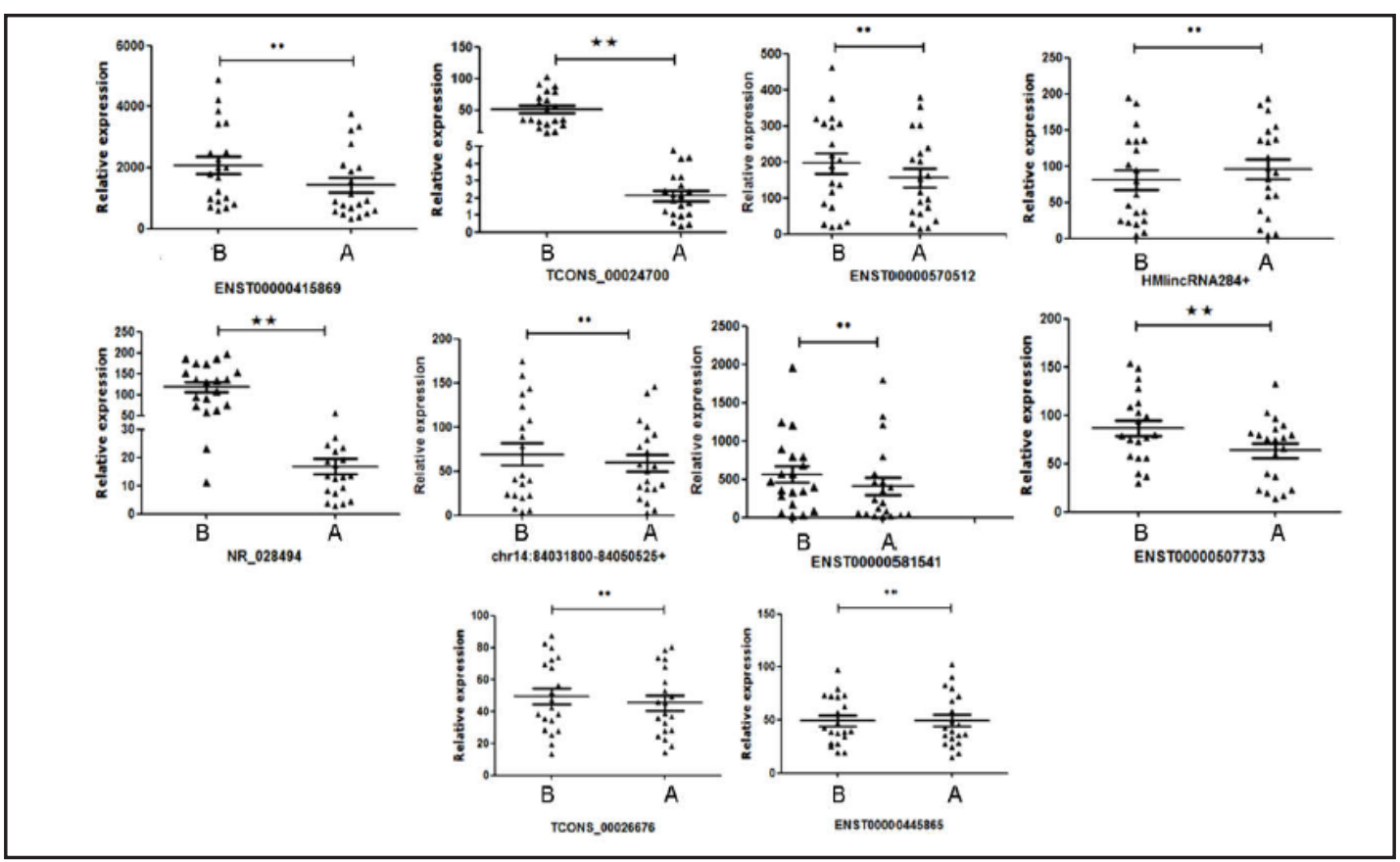

Fig. 4. A total of 20 plasma samples paired between group A and group B were used for validating the expression of 10 downregulated candidate lncRNAs during qRT-PCR analysis. Data are presented as mean \pm SEM. Data was analyzed using Student's t-test. **, $\mathrm{p}<0.05$.

\section{Validation using training and validation sets}

We further examined the differentially expressed lncRNAs by qRT-PCR in a training sample set, including 20 cases with non- ${ }^{131}$ I-avid lung metastasis and 20 with ${ }^{131}$ I-avid lung metastasis (excluding the samples used in the microarray assays). As shown in Fig. 3 and 4, this generated a panel of two IncRNAs (ENST00000462717, ENST00000415582) that were significantly upregulated and three (TCONS_00024700, NR_028494, ENST00000507733) 


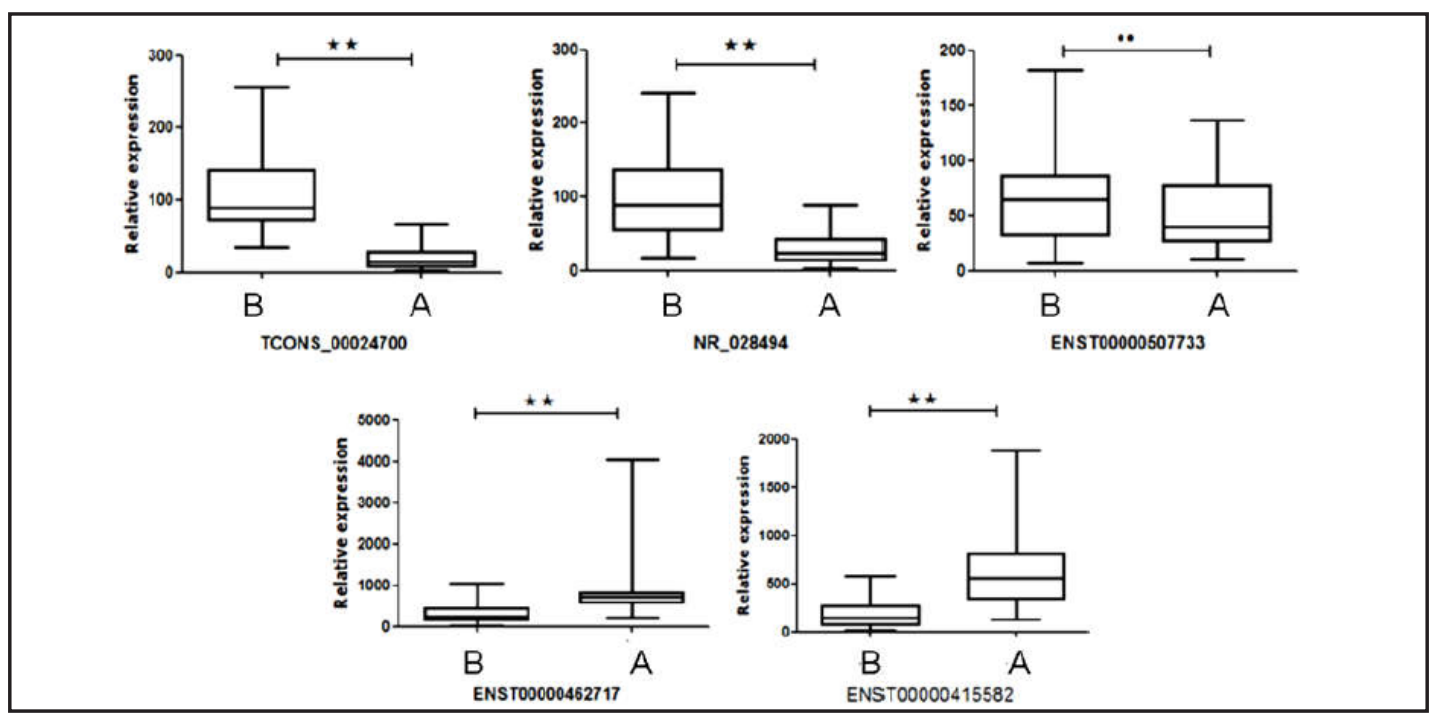

Fig. 5. A total of 46 plasma samples from group A and 108 plasma samples from group B were used for validating the expression of two (ENST00000462717 and ENST00000415582) upregulated and three (TCONS_00024700, NR_028494, and ENST00000507733) downregulated candidate lncRNAs during qRTPCR analysis. Data are presented as mean \pm SEM. Data were analyzed using Student's t-test. **, $p<0.05$.

Fig. 6. The ROC curve displays the relationship of sensitivity and specificity for the four lncRNAs predictive of the ability to concentrate ${ }^{131} \mathrm{I}$ from PTC with lung metastases (A: TCONS_00024700, B: NR_028494, C: ENST 00000462717 , and D: ENST00000415582).
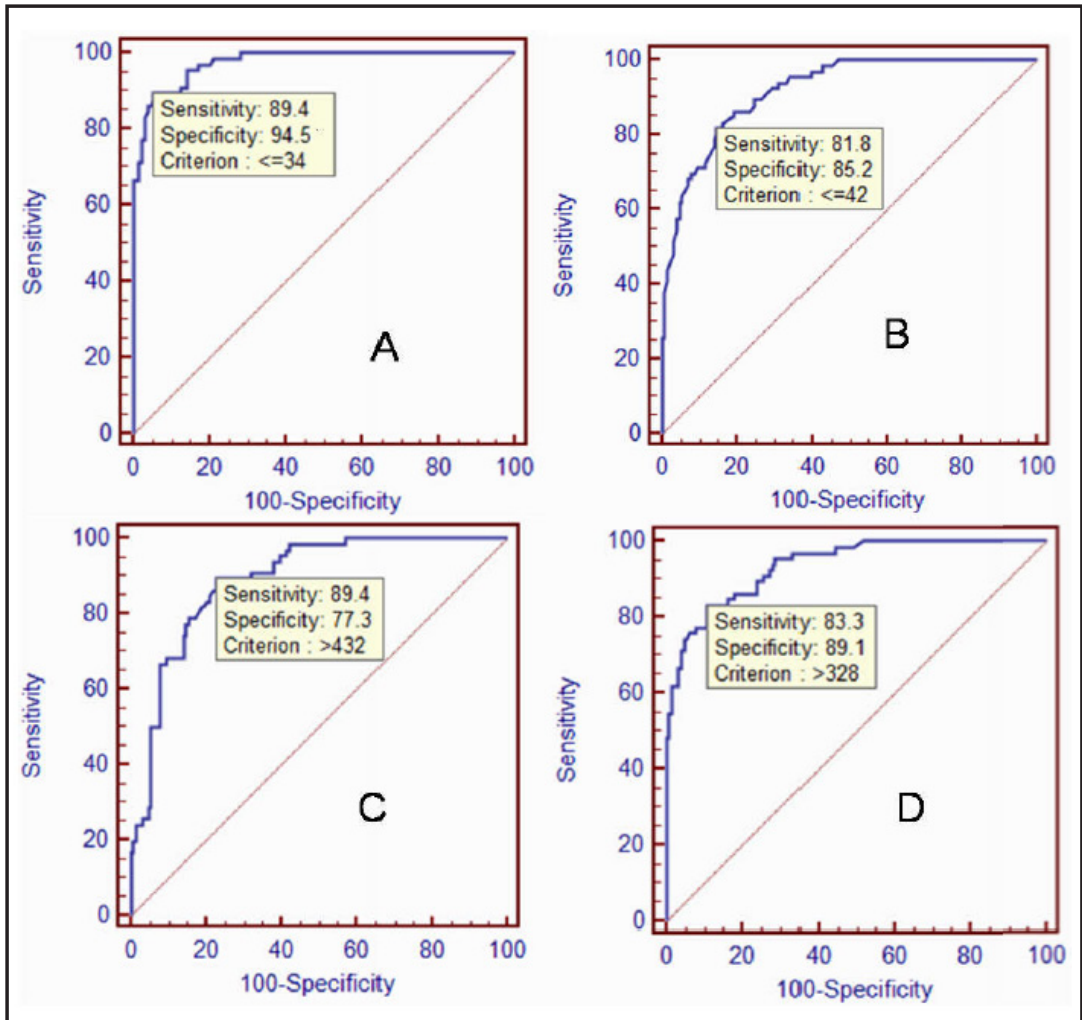

that were significantly downregulated in samples from the non- ${ }^{131} \mathrm{I}-$ avid lung metastasis group $(\mathrm{p}<0.05)$.

To confirm the accuracy and specificity of these five plasma lncRNAs as a potential signature for non- ${ }^{131} \mathrm{I}$-avid lung metastases, we also examined their expression levels in a larger cohort of individual samples (the remaining 46 cases from group A and 108 from group B). As shown in Fig. 5, the expression of two IncRNAs (ENST00000462717, ENST00000415582) 


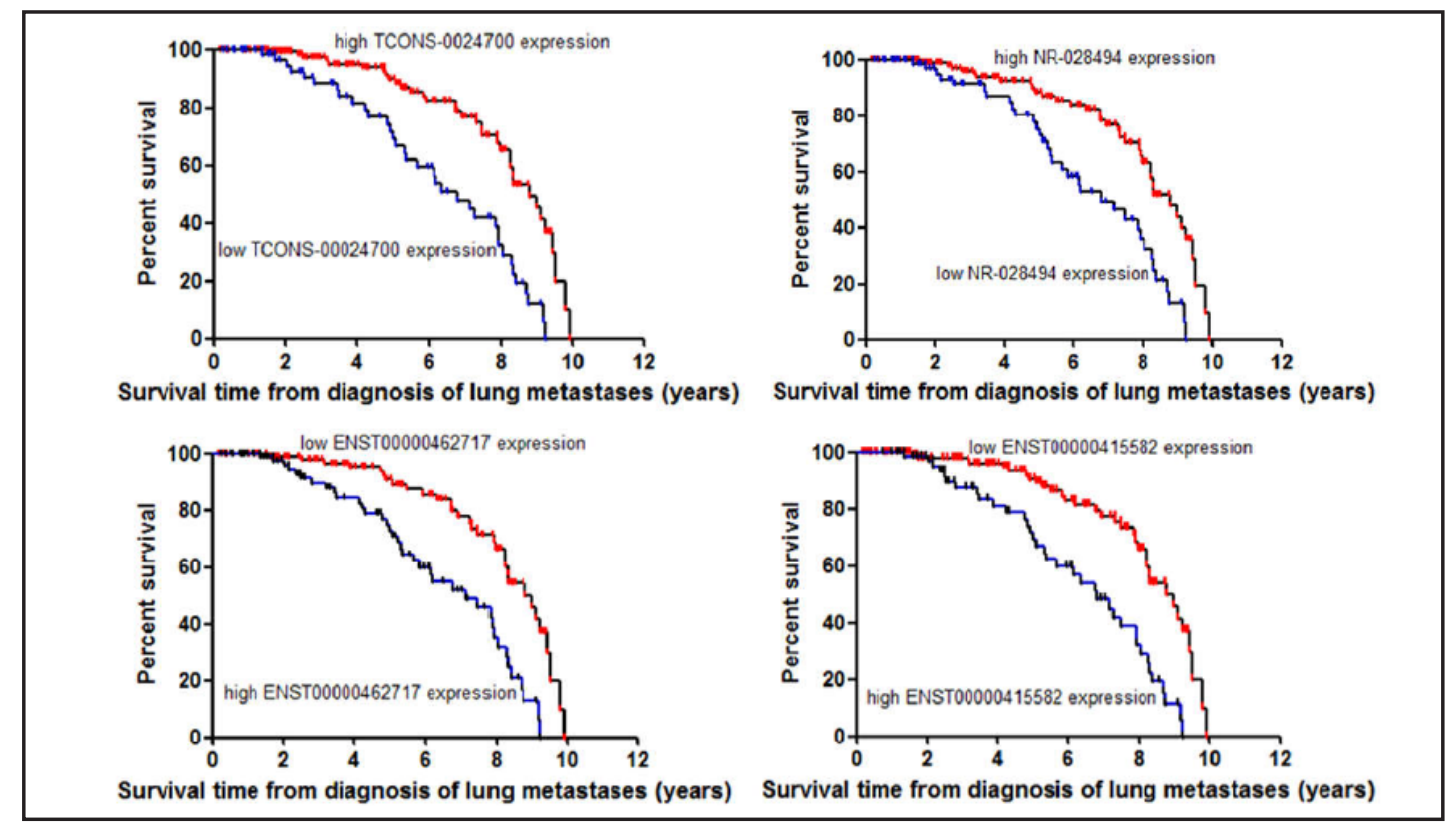

Fig. 7. Kaplan-Meier survival analysis of PTC patients with lung metastases according to the levels of expression of the four IncRNAs. Patients with low ENST00000462717 and ENST00000415582 tended to show better survival than those with high levels; in addition, patients with low levels of TCONS_00024700 and NR_028494 tended to show worse survival than patients with high levels (all $\mathrm{P}<0.001$ ).

in the plasma of patients with non- ${ }^{131}$ I-avid lung metastases was significantly higher than in the ${ }^{131}$ I-avid lung metastases. In addition, the expression of two lncRNAs (TCONS_00024700, NR_028494) in the plasma of patients with non- ${ }^{131}$ I-avid lung metastases was significantly lower than in those with ${ }^{131} \mathrm{I}$-avid lung metastases $(\mathrm{P}<0.05)$. Throughout the multiphase testing and analysis phases, the profile of these four lncRNAs was suggested to be a potential signature for predicting non- ${ }^{131} \mathrm{I}$-avid lung metastases.

Predictive power of four IncRNAs for the ability to concentrate ${ }^{131}$ I of PTC with lung metastasis

To evaluate the predictive power of the four circulating lncRNAs for the ability to concentrate ${ }^{131}$ I of PTC with lung metastases, we performed statistical analysis of the levels of these four lncRNAs in 194 samples (excluding the samples used in the microarray assays). The ROC curve displays the relationship regarding sensitivity and specificity for the four IncRNAs to predict the ability to concentrate ${ }^{131} \mathrm{I}$ in cases with PTC with lung metastases.

ROC analysis of TCONS_00024700 revealed an AUC of 0.975 [95\% confidence interval (CI): 0.942-0.992] (Fig. 6A). The optimal cutoff for TCONS_00024700 was 34, as measured by absolute quantification by qRT-PCR, which had sensitivity of $89.4 \%$ (95\% CI: 79.4\%$95.6 \%)$ and specificity of $94.5 \%$ (95\% CI: 89.1\%-97.8\%) for predicting non- ${ }^{131}$ I-avid lung metastases.

ROC analysis of NR_028494 revealed an AUC of 0.918 (95\% CI: 0.870-0.952) (Fig. 6B). The optimal cutoff for NR_028494 was 42, as measured by absolute quantification by qRTPCR, which had sensitivity of 81.8\% (95\% CI: 70.4\%-90.2\%) and specificity of $85.2 \%$ (95\% CI: 77.8\%-90.8\%) for predicting non- ${ }^{131}$ I-avid lung metastases.

ROC analysis of ENST00000462717 revealed an AUC of 0.890 (95\% CI: 0.837-0.930) (Fig. 6C). The optimal cutoff for ENST00000462717 was 432, as measured by absolute quantification by qRT-PCR, which had sensitivity of $89.4 \%$ (95\% CI: 79.4\%-95.6\%) and specificity of $77.3 \%$ (95\% CI: $69.1 \%-84.3 \%$ ).

ROC analysis of ENST00000415582 revealed an AUC of 0.936 (95\% CI: 0.892-0.966) (Fig. 6D). The optimal cutoff of ENST00000415582 was 328, as measured by absolute 
Fig. 8. Differential expressions of four plasma lncRNAs were showed between effective group (A) and ineffective group (B) for the ${ }^{131}$ I therapy. Data are presented as mean \pm SEM. Data was analyzed using Student's t-test. All $P>0.05$. $P$ value of TCONS_00024700, $\mathrm{N}$ R _ $\begin{array}{llllll}0 & 2 & 8 & 4 & 9 & 4\end{array}$ ENST 00000462717 andENST00000415582 was $0.93,0.11,0.51$ and 0.19 ; respectively.
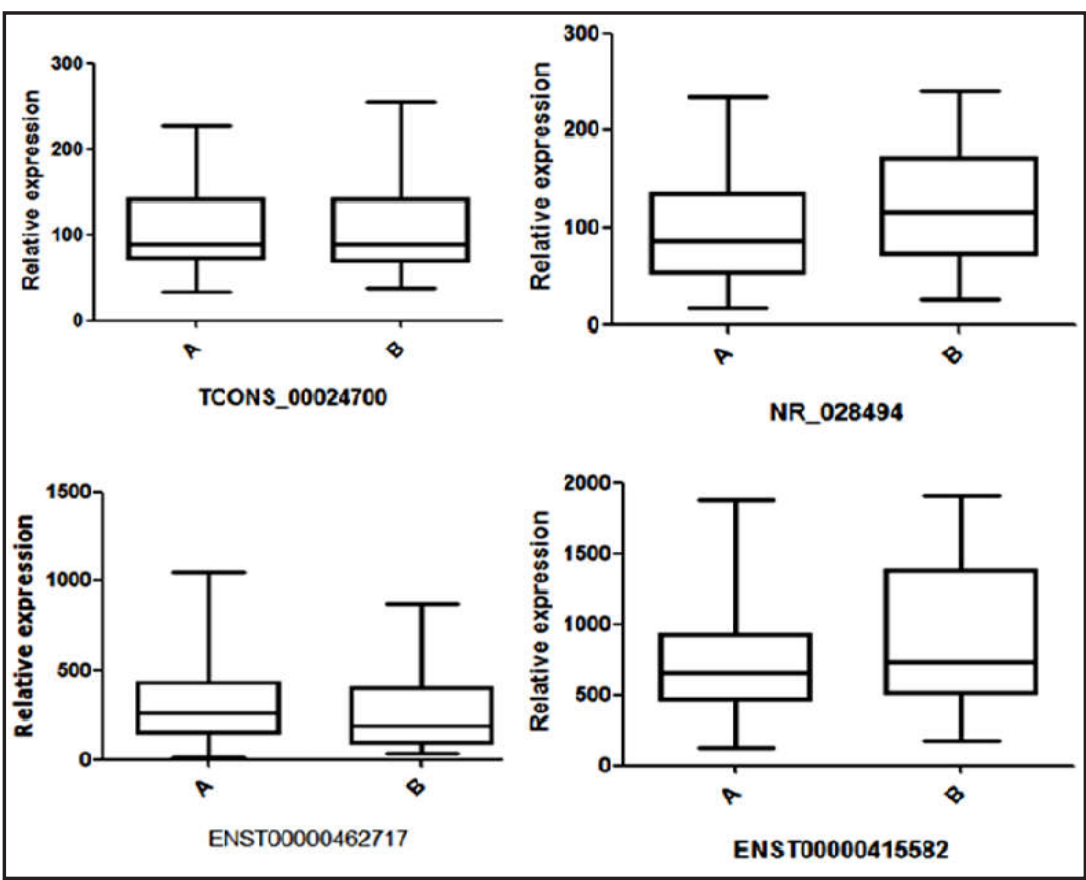

Table. 3. Expression of four circulating lncRNAs for predicting survival in PTC patients with lung metastasis

\begin{tabular}{llllll}
\hline IncRNAS & No. of patients & $\begin{array}{l}\text { No. of } \\
\text { deaths }\end{array}$ & $\begin{array}{l}\text { Ten-year survival rate } \\
{[\%]}\end{array}$ & Log-rank value & P-value \\
\hline $\begin{array}{l}\text { TCONS_00024700 } \\
>34\end{array}$ & 128 & 34 & 73.43 & 20.47 & $<0.001$ \\
$\leq 34$ & 66 & 35 & 46.96 & 11.44 & $<0.001$ \\
NR_028494 & & & & & \\
$>42$ & 121 & 34 & 71.90 & & \\
$\leq 42$ & 73 & 35 & 52.05 & 19.19 & $<0.001$ \\
ENST00000462717 & & & & & \\
$>432$ & 88 & 38 & 56.82 & 12.46 & $<0.001$ \\
$\leq 432$ & 106 & 31 & 70.75 & & \\
ENST00000415582 & & & & & \\
$>328$ & 78 & 33 & 57.64 & & \\
$\leq 328$ & 126 & 36 & 71.42 & & \\
\hline
\end{tabular}

quantification by qRT-PCR, which had sensitivity of $83.3 \%$ (95\% CI: 72.1\%-91.4\%) and specificity of $89.1 \%$ (95\% CI: 82.3\%-93.9\%) for predicting non- ${ }^{131}$ I-avid lung metastases.

\section{Relationship of circulating IncRNA levels with ${ }^{131}$ I therapeutic efficacy in the ${ }^{131}$ I-avid lung} metastases group

Of the 108 lung metastases patients with ${ }^{131}$ I-avid lung metastases of PTC, 21 achieved CR (19.4\%), 45 PR (41.7\%), 23 SD (25.8\%) and 19 had PD (17.6\%). Eighty nine cases (82.4\%) were effective and $19(17.6 \%)$ were ineffective for ${ }^{131}$ I therapy. The expression of the four plasma lncRNAs were compared between two groups according to the efficacy of radioiodine therapy and the results were showed in Fig. 8. The four lncRNAs (TCONS_00024700, NR_028494, ENST00000462717 and ENST00000415582) from patients in effective group and ineffective group were not significantly differentially expressed( all $\mathrm{P}>0.05$ ).

Relationship of circulating lncRNA levels with survival of patients with lung metastases from PTC

Non- ${ }^{131}$ I-avid lung metastases from PTC are often associated with a worse prognosis. We confirmed that the four above mentioned plasma lncRNAs could predict the non- ${ }^{131}$ Iavid lung metastasis of PTC. To determine whether the levels of these four plasma lncRNAs might be indicative of patient prognosis, we performed a comparison of the 10 -year survival 
Fig. 9. Differential expressions of four plasma IncRNAs were showed between lung metastases of PTC patients (A) and healthy individuals (B). All $\mathrm{p}<0.05$. P value of TCONS_00024700, NR_028494, ENST00000462717 and ENST00000415582 was $<0.001,<0.001$, $<0.001$ and 0.035 ,respectively.

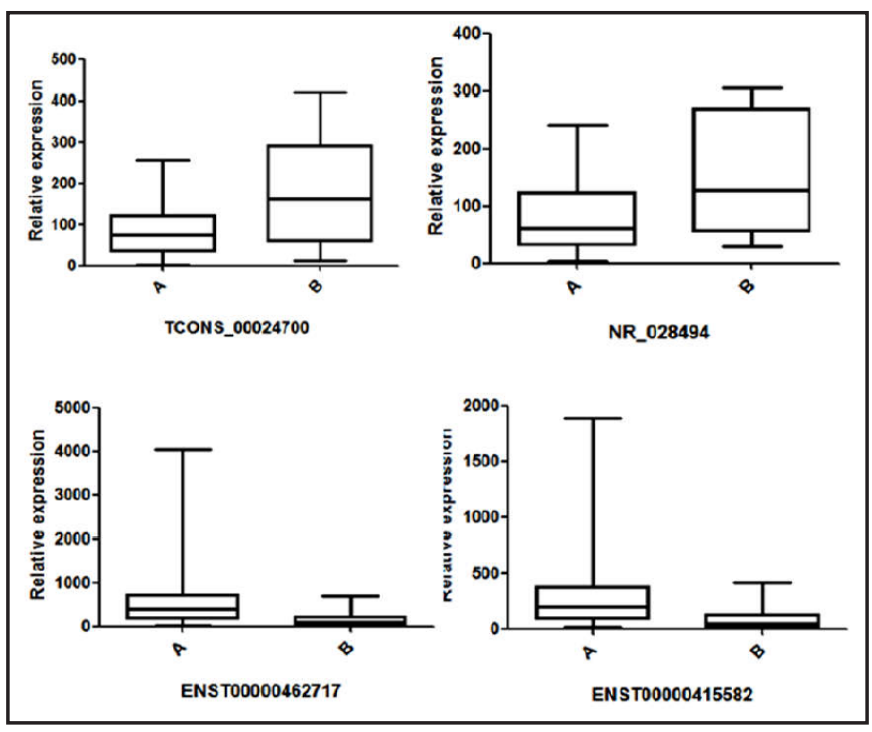

rates between groups with less than (low lncRNAs) and more than the cutoffs of the lncRNAs (high IncRNAs); the number of deaths and 10-year survival rates of these groups are shown in Table 3.

Kaplan-Meier analysis showed that the 10-year survival rates of the low ENST00000462717 and ENST00000415582 groups were $70.75 \%$ and $71.42 \%$, and those of the high ENST00000462717 and ENST00000415582 groups were 56.82\% and $57.64 \%$, respectively. The 10-year survival rates of the low ENST00000462717 and ENST00000415582 groups were significantly different to those of the high ENST00000462717 and ENST00000415582 groups, respectively ( $\mathrm{P}<0.001)$ (Fig. 7).

Kaplan-Meier analysis also showed that the 10-year survival rates of the low TCONS_00024700 and NR_028494 groups were $46.96 \%$ and $52.05 \%$, and those of the high TCONS_00024700 and NR_028494 groups were 73.43\% and 71.09\%, respectively. The 10year survival rates of the low TCONS_00024700 and NR_028494 groups were significantly different to those of the high TCONS_00024700 and NR_028494 groups, respectively $(\mathrm{P}<0.001)$ (Fig. 7).

Comparison of the relative expression of four plasma IncRNAs between PTC patients with lung metastases and healthy individuals

We also detected the expression level of the four IncRNAs in 20 volunteers as healthy control. The expression of the four plasma lncRNAs was compared between PTC patients with lung metastases and healthy individuals (Fig. 9). The expression of two ENST00000462717 and ENST00000415582 in the plasma of patients with lung metastases was significantly higher than those in the healthy individuals $(\mathrm{P}<0.001$ and $\mathrm{P}=0.0035)$ and the expression of TCONS_00024700 and NR_028494 in the plasma of patients with lung metastases was significantly lower than those in the healthy individuals $(\mathrm{P}<0.001)$.

\section{Discussion}

At present, the primary approach to identify non- ${ }^{131}$ I-avid lung metastasis depends on combining the results of ${ }^{131} \mathrm{I}-\mathrm{WBS}$ and serum Tg level. A valuable marker that can be used to predict the ability to concentrate radioiodine in distant metastasis is desirable for changing to a more effective therapeutic regimen that can safely avoid unnecessary radiation and the risk of high serum TSH stimulation.

As far as PTC was concerned, Yang et al. and Lan et al. analyzed lncRNA expression profile by using microarrays in PTC tissues and identified a series of novel PTC-associated 
IncRNAs respectively, which lay the foundation for further investigation of lncRNAs related to PTC $[26,27]$. In addition, important regulatory mechanisms of lncRNAs in the process of tumorigenesis, progression, proliferation, metastases, apoptosis and autophagy of PTC have been reported [27-32]. BRAF-activated IncRNA (BANCR) could activate autophagy [28] and inhibit tumorigenesis in PTC and its levels might be used as a novel prognostic marker [29]. PTC susceptibility candidate 3 (PTCSC3) was found to be a tumor suppressor [30]. IncRNAs (ENST00000426615 and ENST00000537266) might be important regulators of PTC cell proliferation and motility[31]. LncRNA (LOC100507661) expression was elevated in human thyroid cancer tissue and might play a critical role in thyroid carcinogenesis [32]. LncRNA (ANRIL) might reduce $\mathrm{p} 15^{\mathrm{INK} 4 \mathrm{~B}}$ expression through inhibiting TGF- $\beta /$ Smad signaling pathway, promoting invasion and metastasis of thyroid cancer cells, and the silencing of ANRIL inhibited the invasion and metastasis of thyroid cancer cells[33]. With regard to lung metastases of PTC, it was difficult to study the correlation between tissue lncRNAs and ${ }^{131}$ I uptake of lung metastases in PTC patients from the histological level. There were two main reasons as follows: (1) PTC had a favorable prognosis and lung metastases often occurred in the final stage of the PTC patient, therefore, it wasn't easy to find the primary PTC tissue which had been removed from the initial operation [4, 5]; (2) it wasn't convenient and had a little risk to obtain the tissue sample of lung metastatic lesions by CT-guided biopsy.

Liquid biopsy for cancer diagnosis has rapidly developed owing to its noninvasiveness and time-saving procedure $[34,35]$.In the last decade, studies on the utility of circulating nucleic acids such as cell-free DNA, miRNA, and mRNA as novel biomarkers for various human diseases have increased exponentially. Recently, several studies have demonstrated that certain circulating lncRNAs have potential utility as diagnostic markers for numerous cancers and other diseases[17, 20].For example, researchers have identified that three circulating lncRNAs can predict human NSCLC through high-throughput screening[19]. However, to the best of our knowledge, circulating lncRNA profiles haven't been analyzed and compared between non- ${ }^{131}$ I-avid and ${ }^{131}$ I-avid lung metastases from PTC.

In the present study, we thus performed initial screening using microarray assays to measure lncRNA expression followed by extensive qRT-PCR validation between non- ${ }^{131}$ Iavid and ${ }^{131}$ I-avid lung metastases from PTC. We identified a profile in which four lncRNAs (ENST00000462717, ENST00000415582, TCONS_00024700, and NR_028494) differed in their expression in the sera of PTC patients between non- ${ }^{131} \mathrm{I}$-avid and ${ }^{131} \mathrm{I}$-avid lung metastases, including two downregulated lncRNAs (TCONS_00024700 and NR_028494) and two upregulated ones (ENST00000462717 and ENST00000415582). Our results suggest that the use of these four IncRNAs may be a potential minimally invasive biomarker for the preoperative diagnosis of PTC with relatively high sensitivity and specificity. The exosome is a possible vector of the lncRNAs in hematogenous dissemination. However, the mechanisms accounting for the stability of plasma IncRNAs are not well known; they may be protected by exosome encapsulation, as has been shown to occur for plasma miRNAs [36].

For patients with non- ${ }^{131}$ I-avid pulmonary disease of PTC, the benefit of radioiodine therapy was very limited. Loss of ${ }^{131}$ I avidity is associated with a poor outcome. Several studies have reported that DTC patients with ${ }^{131}{ }^{13}$ avidity survive longer than those with non- ${ }^{131}$ I-avid pulmonary metastasis[37, 38]. For example, previously our group showed that PTC patients with non- ${ }^{131}$ I-avid disease had a 10-year survival rate of $38.1 \%$ compared with $69.2 \%$ for those with ${ }^{131} \mathrm{I}$-avid metastases $(\mathrm{P}<0.0001)$ [4]. We confirmed that the four above mentioned plasma lncRNAs might predict the non- ${ }^{131}$ I-avid lung metastasis of PTC. As such, we hypothesized that these four lncRNAs might be related to the prognosis of PTC patients with lung metastases. In view of this, we compared the 10-year survival rates between groups with low and high levels of these lncRNAs. The results confirmed that the levels of these four plasma lncRNAs were associated with the prognosis of PTC patients with lung metastases. Our study also showed that these four IncRNAs weren't associated with the ${ }^{131}$ I therapeutic efficacy in the ${ }^{131} \mathrm{I}$-avid lung metastatic patients.

However, limitations of this study should also be discussed. First, in all of the eligible patients, there was no histopathological verification of the lung metastases. However, 


\section{Cellular Physiology Cell Physiol Biochem 2016;40:1377-1390

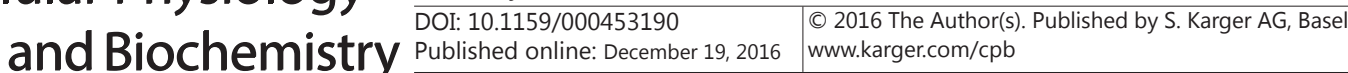 \\ Qiu et al.: Circulating LncRNAs in PTC}

obtaining histological proof of diffuse lesions in lungs was impractical and unethical in these patients. The diagnosis of lung metastasis in the current study was made on the basis of the presence of diffuse bilateral pulmonary nodules, as confirmed by a CT scan, and significantly increased serum Tg levels. Second, although the deregulation of the four IncRNAs in PTC patients with non- ${ }^{131} \mathrm{I}$-avid lung metastatic disease was validated by qRT-PCR, the small sample size in the present study, especially for non-131 I-avid lung metastases and healthy individuals, may make the final results biased. In this regard, further large-scale studies are required to determine the role of circulating lncRNAs in PTC patients with non- ${ }^{131}$ I-avid lung metastases of PTC.

\section{Conclusion}

We identified four IncRNAs, ENST00000462717, ENST00000415582, TCONS_00024700, and NR_028494, that are potential markers for predicting non- ${ }^{131}$ I-avid lung metastatic diseases from PTC. High levels of ENST00000462717 and ENST00000415582 and low levels of TCONS_00024700 and NR_028494 were associated with worse prognosis in PTC patients with lung metastases. These findings provide us with a foundation for the development of a simple, minimally invasive, and effective diagnostic and prognostic tool for the assessment of non- ${ }^{131}$ I-avid lung metastatic diseases from PTC. Long-term followup of the patients in the current study and a prospective study with a larger sample size are needed to further validate the usefulness of circulating lncRNAs in the diagnosis and prognostic assessment of non- ${ }^{131}$ I-avid lung metastatic diseases from PTC.

\section{Acknowledgements}

The authors thank Kang Chen Bio-tech Inc. for their support in the RNA extraction and microarray analyses.

\section{Funding}

This study was sponsored by the National Natural Science Foundation of China (No:81271611 \&81201115).

\section{Disclosure Statement}

The authors declare that there is no conflict of interest that could be perceived as prejudicing the impartiality of the research reported.

\section{References}

1 Siegel RL, Miller KD, Jemal A: Cancer statistics, 2015. CA Cancer J Clin 2015;65:5-29.

2 Pellegriti G, Frasca F, Regalbuto C, Squatrito S, Vigneri R: Worldwide increasing incidence of thyroid cancer: Update on epidemiology and risk factors. J Cancer Epidemiol 2013;2013:965212.

3 Brenner H: Long-term survival rates of cancer patients achieved by the end of the 20th century: A period analysis. Lancet 2002;360:1131-1135.

4 Song HJ, Qiu ZL, Shen CT, Wei WJ, Luo QY: Pulmonary metastases in differentiated thyroid cancer: Efficacy of radioiodine therapy and prognostic factors. Eur J Endocrinol 2015;173:399-408.

5 Qiu ZL, Song HJ, Xu YH, Luo QY: Efficacy and survival analysis of 131i therapy for bone metastases from differentiated thyroid cancer. J Clin Endocrinol Metab 2011;96:3078-3086. 


\section{Cellular Physiology Cell Physiol Biochem 2016;40:1377-1390 \begin{tabular}{l|l} 
and Biochemistry Published online: December 19, 2016 & $\begin{array}{l}\text { C } 2016 \text { The Author(s). Published by S. Karger AG, Basel } \\
\text { www.karger.com/cpb }\end{array}$
\end{tabular} \\ Qiu et al.: Circulating LncRNAs in PTC}

6 Casara D, Rubello D, Saladini G, Masarotto G, Favero A, Girelli ME, Busnardo B: Different features of pulmonary metastases in differentiated thyroid cancer: Natural history and multivariate statistical analysis of prognostic variables. J Nucl Med 1993;34:1626-1631.

7 Esteller M: Non-coding rnas in human disease. Nat Rev Genet 2011;12:861-874.

8 Costa FF: Non-coding rnas: Meet thy masters. Bioessays 2010;32:599-608.

9 Zhang R, Hardin H, Chen J, Guo Z, Lloyd RV: Non-coding rnas in thyroid cancer. Endocr Pathol 2016;27:1220.

10 Luo G, Wang M, Wu X, Tao D, Xiao X, Wang L, Min F, Zeng F, Jiang G: Long non-coding rna meg3 inhibits cell proliferation and induces apoptosis in prostate cancer. Cell Physiol Biochem 2015;37:2209-2220.

11 Li J, Wang X, Tang J, Jiang R, Zhang W, Ji J, Sun B: Hulc and linc00152 act as novel biomarkers in predicting diagnosis of hepatocellular carcinoma. Cell Physiol Biochem 2015;37:687-696.

12 Zhou X, Ye F, Yin C, Zhuang Y, Yue G, Zhang G: The interaction between mir-141 and lncrna-h19 in regulating cell proliferation and migration in gastric cancer. Cell Physiol Biochem 2015;36:1440-1452.

13 Li C, Chen J, Zhang K, Feng B, Wang R, Chen L: Progress and prospects of long noncoding rnas (lncrnas) in hepatocellular carcinoma. Cell Physiol Biochem 2015;36:423-434.

14 Yang ZT, Li Z, Wang XG, Tan T, Yi F, Zhu H, Zhao JP, Zhou XF: Overexpression of long non-coding rna zxf2 promotes lung adenocarcinoma progression through c-myc pathway. Cell Physiol Biochem 2015;35:23602370.

15 Wang Y, Gao S, Liu G, Jia R, Fan D, Feng X: Microarray expression profile analysis of long non-coding rnas in human gastric cardiac adenocarcinoma. Cell Physiol Biochem 2014;33:1225-1238.

16 Arita T, Ichikawa D, Konishi H, Komatsu S, Shiozaki A, Shoda K, Kawaguchi T, Hirajima S, Nagata H, Kubota T, Fujiwara H, Okamoto K, Otsuji E: Circulating long non-coding rnas in plasma of patients with gastric cancer. Anticancer Res 2013;33:3185-3193.

17 Tinzl M, Marberger M, Horvath S, Chypre C: Dd3pca3 rna analysis in urine--a new perspective for detecting prostate cancer. Eur Urol 2004;46:182-186; discussion 187.

18 Isin M, Ozgur E, Cetin G, Erten N, Aktan M, Gezer U, Dalay N: Investigation of circulating lncrnas in b-cell neoplasms. Clin Chim Acta 2014;431:255-259.

19 Tang Q, Ni Z, Cheng Z, Xu J, Yu H, Yin P: Three circulating long non-coding rnas act as biomarkers for predicting nsclc. Cell Physiol Biochem 2015;37:1002-1009.

20 Shi J, Li X, Zhang F, Zhang C, Guan Q, Cao X, Zhu W, Zhang X, Cheng Y, Ou K, Chen Q, Hu S: Circulating Incrnas associated with occurrence of colorectal cancer progression. Am J Cancer Res 2015;5:2258-2265.

21 Fayda M, Isin M, Tambas M, Guveli M, Meral R, Altun M, Sahin D, Ozkan G, Sanli Y, Isin H, Ozgur E, Gezer U: Do circulating long non-coding rnas (lncrnas) (lincrna-p21, gas 5, hotair) predict the treatment response in patients with head and neck cancer treated with chemoradiotherapy? Tumour Biol 2016;37:3969-3978.

22 Fritah S, Niclou SP, Azuaje F: Databases for lncrnas: A comparative evaluation of emerging tools. RNA 2014;20:1655-1665.

23 Chakraborty S, Deb A, Maji RK, Saha S, Ghosh Z: Lncrbase: An enriched resource for Incrna information. PLoS One 2014;9:e108010.

24 Quek XC, Thomson DW, Maag JL, Bartonicek N, Signal B, Clark MB, Gloss BS, Dinger ME: Lncrnadb v2.0: Expanding the reference database for functional long noncoding rnas. Nucleic Acids Res 2015;43:D168173.

25 Nishino M, Jagannathan JP, Ramaiya NH, Van den Abbeele AD: Revised recist guideline version 1.1: What oncologists want to know and what radiologists need to know. AJR Am J Roentgenol 2010;195:281-289.

26 Yang M, Tian J, Guo X, Yang Y, Guan R, Qiu M, Li Y, Sun X, Zhen Y, Zhang Y, Chen C, Li Y, Fang H: Long noncoding rna are aberrantly expressed in human papillary thyroid carcinoma. Oncol Lett 2016;12:544552.

27 Lan X, Zhang H, Wang Z, Dong W, Sun W, Shao L, Zhang T, Zhang D: Genome-wide analysis of long noncoding rna expression profile in papillary thyroid carcinoma. Gene 2015;569:109-117.

28 Wang Y, Guo Q Zhao Y, Chen J, Wang S, Hu J, Sun Y: Braf-activated long non-coding rna contributes to cell proliferation and activates autophagy in papillary thyroid carcinoma. Oncol Lett 2014;8:1947-1952.

29 Liao T, Qu N, Shi RL, Guo K, Ma B, Cao YM, Xiang J, Lu ZW, Zhu YX, Li DS, Ji QH: Braf-activated lncrna functions as a tumor suppressor in papillary thyroid cancer. Oncotarget DOI:10.18632/oncotarget.10825.

30 Fan M, Li X, Jiang W, Huang Y, Li J, Wang Z: A long non-coding rna, ptcsc3, as a tumor suppressor and a target of mirnas in thyroid cancer cells. Exp Ther Med 2013;5:1143-1146. 


\section{Cellular Physiology Cell Physiol Biochem 2016;40:1377-1390 \begin{tabular}{ll|l} 
DOI: 10.1159/000453190 & $\begin{array}{l}\text { O 2016 The Author(s). Published by S. Karger AG, Basel } \\
\text { www.karger.com/cpb }\end{array}$
\end{tabular} \\ Qiu et al.: Circulating LncRNAs in PTC}

31 Xu B, Shao Q, Xie K, Zhang Y, Dong T, Xia Y, Tang W: The long non-coding rna enst00000537266 and enst00000426615 influence papillary thyroid cancer cell proliferation and motility. Cell Physiol Biochem 2016;38:368-378.

32 Kim D, Lee WK, Jeong S, Seol MY, Kim H, Kim KS, Lee EJ, Lee J, Jo YS: Upregulation of long noncoding rna loc100507661 promotes tumor aggressiveness in thyroid cancer. Mol Cell Endocrinol 2016;431:36-45.

33 Zhao JJ, Hao S, Wang LL, Hu CY, Zhang S, Guo LJ, Zhang G, Gao B, Jiang Y, Tian WG, Luo DL: Long non-coding rna anril promotes the invasion and metastasis of thyroid cancer cells through tgf-beta/smad signaling pathway. Oncotarget DOI:10.18632/oncotarget.11087.

34 Crowley E, Di Nicolantonio F, Loupakis F, Bardelli A: Liquid biopsy: Monitoring cancer-genetics in the blood. Nat Rev Clin Oncol 2013;10:472-484.

35 Pantel K, Alix-Panabieres C: Real-time liquid biopsy in cancer patients: Fact or fiction? Cancer Res 2013;73:6384-6388.

36 Senfter D, Holzner S, Kalipciyan M, Staribacher A, Walzl A, Huttary N, Krieger S, Brenner S, Jager W, Krupitza G, Dolznig H, Mader RM: Loss of mir-200 family in 5-fluorouracil resistant colon cancer drives lymphendothelial invasiveness in vitro. Hum Mol Genet 2015;24:3689-3698.

37 Cho SW, Choi HS, Yeom GJ, Lim JA, Moon JH, Park DJ, Chung JK, Cho BY, Yi KH, Park YJ: Long-term prognosis of differentiated thyroid cancer with lung metastasis in korea and its prognostic factors. Thyroid 2014;24:277-286.

38 Pelizzo MR, Boschin IM, Toniato A, Piotto A, Pagetta C, Gross MD, Al-Nahhas A, Rubello D: Papillary thyroid carcinoma: 35-year outcome and prognostic factors in 1858 patients. Clin Nucl Med 2007;32:440-444. 